Setting a New Standard in the Analysis of Binary Stars

K. Pavlovski, A. Tkachenko and G. Torres (eds)

EAS Publications Series, 64 (2013) 237-244

\title{
LEAST-SQUARES DECONVOLUTION BASED ANALYSIS OF STELLAR SPECTRA
}

\author{
T. Van Reeth ${ }^{1}$, A. Tkachenko ${ }^{1}$ and V. Tsymbal ${ }^{2}$
}

\begin{abstract}
In recent years, astronomical photometry has been revolutionised by space missions such as MOST, CoRoT and Kepler. However, despite this progress, high-quality spectroscopy is still required as well. Unfortunately, high-resolution spectra can only be obtained using ground-based telescopes, and since many interesting targets are rather faint, the spectra often have a relatively low $\mathrm{S} / \mathrm{N}$. Consequently, we have developed an algorithm based on the least-squares deconvolution profile, which allows to reconstruct an observed spectrum, but with a higher $\mathrm{S} / \mathrm{N}$. We have successfully tested the method using both synthetic and observed data, and in combination with several common spectroscopic applications, such as e.g. the determination of atmospheric parameter values, and frequency analysis and mode identification of stellar pulsations.
\end{abstract}

\section{Introduction}

Thanks to space missions such as MOST, CoRoT and Kepler, there have been many advances in the field of photometry over the last decade. However, astronomical research still fundamentally depends on spectroscopy as well. On one hand, it allows us to, e.g., obtain values for the fundamental stellar parameters, while on the other, time series of spectra with a relatively high $\mathrm{S} / \mathrm{N}$ often allow us to analyse stellar variability or binarity. It is also for this reason that, in the case of double-lined spectroscopic binaries, disentangling composite spectra is such a valuable technique: it allows us to gain detailed information about the individual components, which is nigh-on impossible for photometric data. Unfortunately, we currently need ground-based observations to obtain high-resolution spectra, and since many of the interesting objects are faint, ofttimes $4 \mathrm{~m}$-class telescopes are required to properly observe targets with a high $\mathrm{S} / \mathrm{N}$. However, as observation time

\footnotetext{
${ }^{1}$ Instituut voor Sterrenkunde, KU Leuven, Celestijnenlaan 200D, 3001 Leuven, Belgium

2 Tavrian National University, Department of Astronomy, Simferopol, Ukraine
} 
on these instruments is limited, observers often have to make due with $2 \mathrm{~m}$-class telescopes, resulting in lower $\mathrm{S} / \mathrm{N}$ data.

With this shortcoming in mind, we developed a new algorithm, based on the least-squares deconvolution (LSD) profile, with the aim to filter out noise of observed high-resolution spectra. In these proceedings we only provide a concise description of the algorithm and several of the conducted tests, and we refer the interested reader to Tkachenko et al. (2013) for more detailed information.

\section{The Least-Squares Deconvolution algorithm}

\subsection{The classical LSD profile}

The basic idea of the LSD technique is to extract an "average" high-precision line profile from stellar intensity or polarisation spectra. The method was first proposed and developed by Donati et al. (1997), and is based on two assumptions: (i) individual spectral lines have the same intrinsic line profile, and (ii) overlapping spectral lines add up linearly. We can therefore write the spectrum $Y$ as

$$
Y=M * Z(v),
$$

where $M=\sum_{i} w_{i} \delta\left(v-v_{i}\right)$ is the line pattern function (with $w_{i}$ the central line depths of the individual lines) and $Z(v)$ is the intrinsic line profile. As described by Donati et al. (1997) and Kochukhov et al. (2010), this equation can be rewritten using matrices

$$
\mathbf{Y}=\mathbf{M} \cdot \mathbf{Z}
$$

which in turn can be expressed as the inverse problem

$$
\mathbf{Z}=\left(\mathbf{M}^{T} \cdot \mathbf{S}^{2} \cdot \mathbf{M}\right)^{-1} \cdot\left(\mathbf{M}^{T} \cdot \mathbf{S}^{2} \cdot \mathbf{Y}_{0}\right) .
$$

Here $\mathbf{Y}_{0}$ is the observed spectrum and $\mathbf{S}^{2}$ is a diagonal weighting matrix based on the variance of the data points in the spectrum. We note that, by solving for the LSD profile $\mathbf{Z}$, we effectively deconvolve the weighted cross-correlation vector with the auto-correlation matrix. This allows us to remove the spurious signal which might be present in the cross-correlation due to regularities in the used line pattern function.

Unfortunately, the multiplication with the numerically computed inverse of the matrix also tends to amplify the noise which is present in the cross-correlation. To avoid this complication, we chose to solve the inverse problem using the LevenbergMarquardt algorithm. We do not make any assumptions beforehand on either the shape or the depth of the LSD-profile, but take the continuum flux level at unity as the initial guess for the profile. And in order to limit the computation time, we use a fast modified version of the Levenberg-Marquardt algorithm (developed by Piskunov \& Kochukhov 2002). 


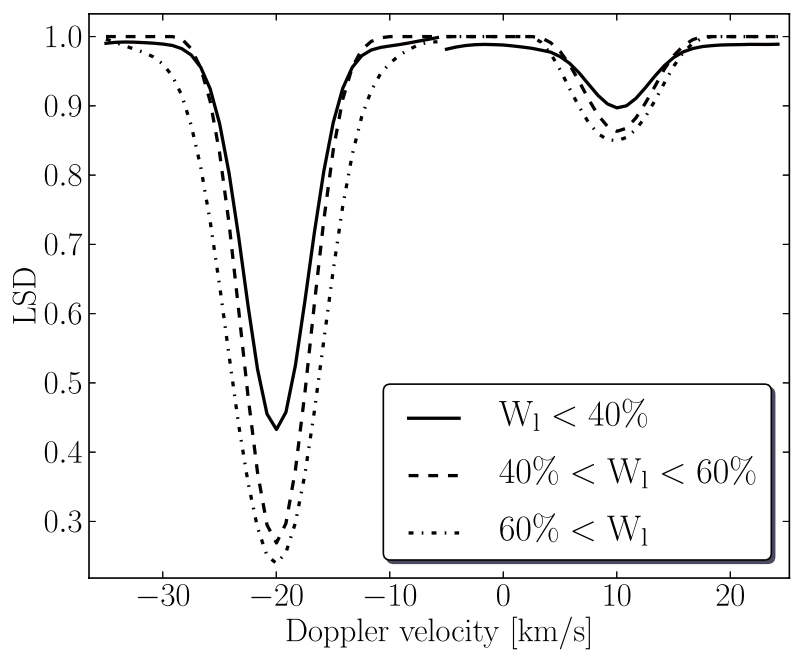

Fig. 1. The LSD profiles computed for a synthetic composite spectrum of a binary $\left(L_{1} / L_{\text {tot }}=85 \%\right.$; primary: $T_{\text {eff }}=8500 \mathrm{~K}, \log g=3.4 \operatorname{dex},[\mathrm{M} / \mathrm{H}]=0.0 \mathrm{dex}$, $v \sin i=2 \mathrm{~km} \mathrm{~s}^{-1} ;$ secondary: $T_{\text {eff }}=7300 \mathrm{~K}, \log g=3.5 \mathrm{dex},[\mathrm{M} / \mathrm{H}]=-0.3 \mathrm{dex}$, $\left.v \sin i=2 \mathrm{~km} \mathrm{~s}^{-1}\right)$. The line strength $w_{l}$ is expressed here as a percentage of the continuum flux.

\subsection{Generalisation of the technique}

\subsubsection{The multi-profile technique}

Aside from the numerical calculations, the LSD algorithm itself can also be improved. The intrinsic line shape, for instance, actually varies from line to line, e.g. dependent on the pressure broadening. To take this into account, Kochukhov et al. (2010) assumed each spectral line to be a linear combination of different intrinsic line shapes, each of which corresponds to a different line pattern function and can be located in different parts of the velocity space:

$$
Y=\sum_{j} M_{j} * Z_{j}(v)
$$

This allows us, for instance, to properly apply the LSD algorithm on composite spectra of an SB2 consisting of components with different atmospheric parameter values. And by computing different LSD profiles for the spectral lines of a single star, in function of the line depths, we can also partially take into account variations in the pressure broadening of spectral lines. (See Fig. 1) Unfortunately, the multi-profile implementation of the LSD technique is still insufficient to model hydrogen, helium, and strong metal lines with dampened wings. As a consequence, our generalised LSD technique is only applicable for stellar spectra with a large 


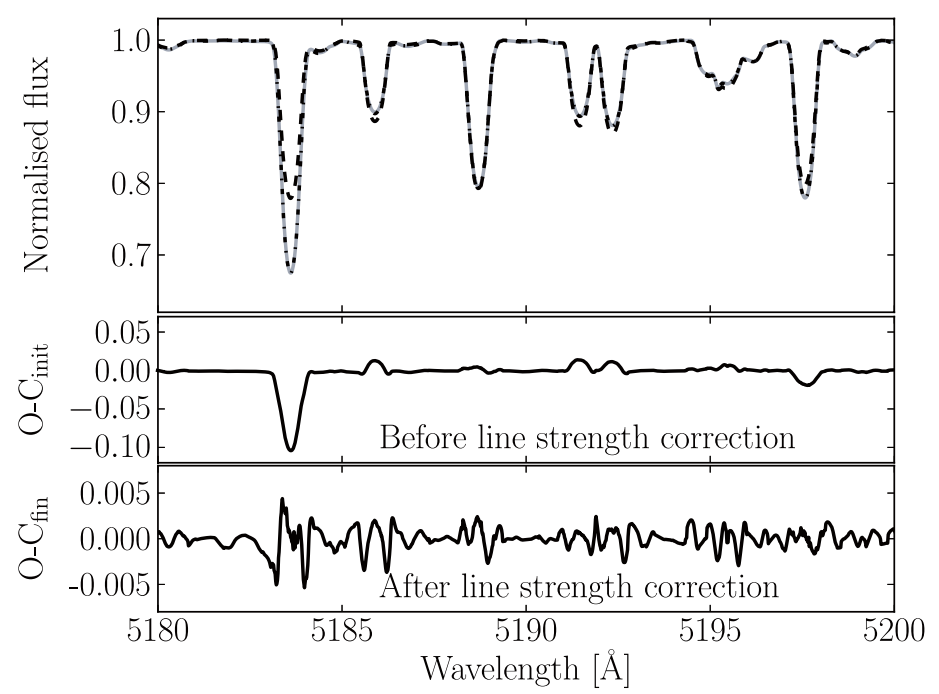

Fig. 2. The spectral reconstruction for a synthetic spectrum $\left(T_{\text {eff }}=8500 \mathrm{~K}, \log g=\right.$ $3.4 \mathrm{dex},[\mathrm{M} / \mathrm{H}]=0.0 \mathrm{dex}, \xi=2 \mathrm{~km} \mathrm{~s}^{-1}$ and $\left.v \sin i=20 \mathrm{~km} \mathrm{~s}^{-1}\right)$. The upper plot shows the input spectrum (grey full line), and the LSD reconstructions before (dashed) and after (dash-dotted) the line strength adaptation. Note also the scaling of the axes for the $\mathrm{O}-\mathrm{C}$ data.

number of usable metal lines. So far the algorithm has been tested for stars with a $T_{\text {eff }}$ between $5000 \mathrm{~K}$ and $10000 \mathrm{~K}$. The performance of the code for other stars is unknown.

\subsubsection{Line strength correction}

If we now compute the convolution of the obtained LSD profiles and the lines in the line pattern functions, we should be able to reconstruct the observed spectrum, but with a much higher $\mathrm{S} / \mathrm{N}$ (as we can see from Eq. (2.1)). However, many spectral lines in the observed spectrum are likely to have central line depths which differ from the values used in the line pattern function. In addition, we incorrectly assumed that blended lines add up linearly (see Sect. 2.1): e.g. line blending due to pressure broadening of the line profiles is non-linear. As a consequence, there will be discrepancies between the observed and reconstructed spectrum. In order to compensate for these shortcomings, we "optimise" the spectral line strengths by fitting the reconstructed spectrum to the observed one. (See Fig. 2). The strengths of the spectral lines are adapted one at a time (by means of the golden section search algorithm), taking into account the contributions from the other lines. If, however, neighbouring spectral lines cannot be resolved by the used spectrograph, we do not treat them separately, but solve for the sum of their line strengths instead. 
To allow all of the line strengths to properly converge towards the solution, we iterate several times over the complete line list (typically ten times). Afterwards, we recompute the LSD profiles, and iterate globally over all the computations, until neither the LSD profiles nor the line pattern functions vary significantly anymore. Typically, two global iterations of the entire computation cycle is sufficient.

\section{Applications}

While the original goal of this research was mainly to analyse to binaries, we first tested the algorithm on both synthetic and observed spectra of single stars, before taking into account the effect of binarity. Both the synthetic spectra and the line masks (i.e., the line pattern functions) used in these tests were computed with the SYNTHV code (Tsymbal 1996). The atmosphere models we used in the SYNTHV code were computed with the LLMODELS code (Shulyak et al. 2004), based on information extracted from the VALD database (Kupka et al. 1999).

\subsection{Single stars}

\subsubsection{Atmospheric parameter value determination}

The first application test we conducted was the determination of atmospheric parameter values, for both synthetic and observational data. In both cases, the parameter value computations were done using the GSSP code (Lehmann et al. 2011). For the run with the synthetic spectra we took $T_{\text {eff }}=8500 \mathrm{~K}, \log g=$ $3.4 \mathrm{dex},[\mathrm{M} / \mathrm{H}]=0.0 \mathrm{dex}, \xi=2 \mathrm{~km} \mathrm{~s}^{-1}$ and $v \sin i=2 \mathrm{~km} \mathrm{~s}^{-1}$ and added varying degrees of noise $(\mathrm{S} / \mathrm{N}=35 ; 70 ; \infty)$, while the atmospheric parameters of the line masks varied near those of the input spectra $\left(T_{\text {eff }} \pm 500 \mathrm{~K}, \log g \pm 0.5 \mathrm{dex}\right.$, $[\mathrm{M} / \mathrm{H}] \pm 0.3 \mathrm{dex})$. By comparing the atmospheric parameter values computed from the LSD reconstructions with the ones from the synthetic input spectra, we were able to also study the precise influence of the chosen line pattern function on the end result. The test results then showed that the differences between the LSD reconstructed parameter values and those of the input spectrum were systematically smaller than the error margins (obtained using $\chi^{2}$ statistics). The largest deviations were obtained when the metallicity of the used mask differed from the metallicity of the input spectrum.

The application of the LSD algorithm on observational data provided similar results. We used spectra of Vega $\left(v \sin i=22 \mathrm{~km} \mathrm{~s}^{-1}, \mathrm{~S} / \mathrm{N} \sim 100\right)$ and KIC 4749989 $\left(v \sin i=190 \mathrm{~km} \mathrm{~s}^{-1}, \mathrm{~S} / \mathrm{N} \sim 60\right)$, taken with the HERMES spectrograph at the $1.2 \mathrm{~m}$ Mercator Telescope (Observatorio del Roque de los Muchachos, La Palma). As illustrated in Figure 3, the observed spectra were reconstructed very well, and the atmospheric parameter values computed from these LSD reconstructions agreed with the values found in the literature within the $1 \sigma$ confidence level. 


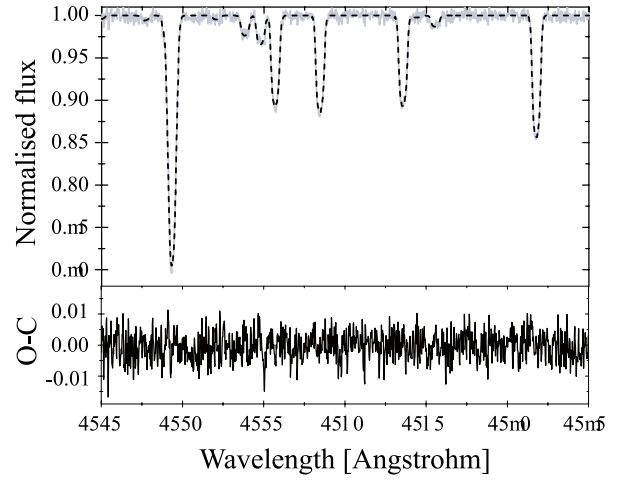

(a) for Vega

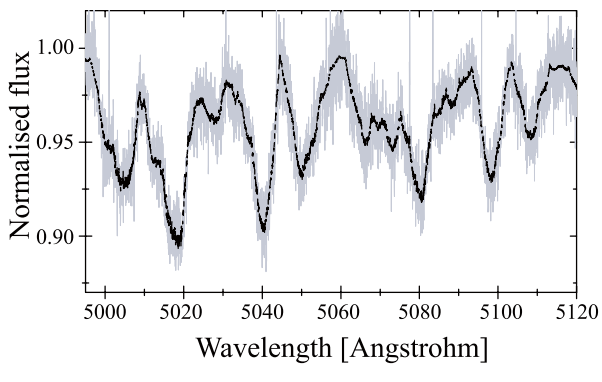

(b) for KIC 4749989

Fig. 3. A comparison of the observed (grey) and LSD reconstructed (black) spectra.

\subsubsection{Intrinsically variable stars}

Next, we applied the LSD algorithm on stellar spectra showing intrinsic line profile variations due to pulsations. The first test case was $20 \mathrm{CVn}$, a monoperiodic radial $\delta$ Scuti pulsator (e.g., Rodriguez et al. 1998). The spectra were retrieved from the archive of the high-resolution échelle spectrograph ELODIE $(R=$ 42000,67 orders, $3895-6815 \AA$ ) at the $193 \mathrm{~cm}$-telescope at the Observatoire de Haute-Provence (OHP), which was in use from 1993 to 2006. We worked with the ScII-line at $5239 \AA$, and by adding gaussian white noise to the observed data (S/N $\sim 40$ ) before applying the LSD algorithm, we saw that, while the quality of the noisy "original" data was insufficient to do either a frequency analysis or pulsational mode identification, the time series of LSD reconstructed ScII-lines showed no such limitations. For our other test case, HD 189631, a multi-periodic nonradial $\gamma$ Doradus pulsator, we obtained equally successful results as we did for $20 \mathrm{CVn}$.

These tests showed that, if the spectral lines show the same response to the pulsations and assuming we have enough observations to resolve the oscillation frequencies, we can determine the oscillation frequency values and do a mode identification $^{3}$ from either the LSD profiles themselves, or from a (properly) reconstructed non-blended spectral line (Zima 2008).

\subsection{Binary stars}

Thanks to the multi-profile implementation of the LSD technique, we are also able to properly take into account binarity, which allows us to facilitate the analysis of noisy composite spectra of SB2s. A good example is KIC 11285625, a double-lined

\footnotetext{
${ }^{3}$ Mode identification results obtained with the software package FAMIAS developed in the framework of the FP6 European Coordination Action HELAS (http://www.eu-helas.org/).
} 
binary where the primary shows $\gamma$ Dor-type pulsations (Debosscher et al. 2013). In the original paper, the authors obtained the disentangled spectra of both binary components by means of the FDBINARY code (Ilijić et al. 2004), but the spectrum of the secondary was of too low $\mathrm{S} / \mathrm{N}$ the atmospheric parameters determination to be possible. Tkachenko et al. (2013) showed that application of our LSD technique to the original composite spectra and application of the spectral disentangling to the LSD model spectra afterwards leads to significant increase in $\mathrm{S} / \mathrm{N}$ of both decomposed spectra providing a good enough quality of the data to make detailed spectrum analysis of both binary components possible.

\subsubsection{Spectral disentangling}

The analysis by Debosscher et al. (2013) again confirms the usefulness of spectral disentangling. It is therefore also worth noting that it is possible to use the LSD algorithm to do spectral disentangling: we only have to make sure that, when we convolve the LSD profiles and the line pattern functions in Equation (2.1), we do this for each contributing stellar component individually. Unfortunately however, the spectral line strength correction which is applied afterwards, also introduces a degeneracy in the solution, since most of the spectral lines of the primary and the secondary overlap. As a result, proper spectral disentangling based on the LSD algorithm is currently not yet possible, though we have several ideas on how to solve this problem.

\subsubsection{Light factor estimation}

Applying the LSD algorithm to the composite spectra of SB2s, can further provide us with an estimate of the light factor $\alpha$ of the system, if we assume that

$$
\alpha=\frac{E W_{1}^{L S D}}{E W_{1}^{L S D}+E W_{2}^{L S D}} .
$$

We tested this hypothesis by taking a synthetic composite binary spectrum with the same atmospheric parameters as for the LSD profiles in Figure 1 (primary: $T_{\text {eff }}=8500 \mathrm{~K}, \log g=3.4 \mathrm{dex},[\mathrm{M} / \mathrm{H}]=0.0 \mathrm{dex} ;$ secondary: $T_{\text {eff }}=7300 \mathrm{~K}, \log g=$ $3.5 \mathrm{dex},[\mathrm{M} / \mathrm{H}]=-0.3 \mathrm{dex}$ ), and then allowing the atmospheric parameter values of the used line masks to vary (similar to the tests we conducted in Sect. 3.1.1) to test the influence of the used line masks.

In most cases the light factor $\alpha$ could be recovered with an accuracy of about $5 \%$ of the continuum. Though there is an exception when the metallicities of the used line masks differs from the metallicities of the input spectrum: then the error of the recovered light factor can go up to about $12 \%$.

\section{Conclusions \& future work}

With the launches of high-performance space missions, astronomical research based on photometry has been revolutionised. However, we still require spectroscopic observations as well, since a lot of useful information, such as e.g. fundamental stellar 
parameter values cannot be determined from the analysis of white-light photometric light curves. Unfortunately, many interesting targets are faint, and due to a limited observation time, of times $2 \mathrm{~m}$-class telescopes have to be used, resulting in rather noisy spectra. We have developed an algorithm, based on the LSD technique, which allows us to reconstruct stellar spectra of both single and binary stars, but with a much higher S/N. So far the method has been tested for stars with $5000 \mathrm{~K} \leq T_{\text {eff }} \leq 10000 \mathrm{~K}$, and has been shown to be compatible with several other spectral analysis techniques, including the determination of atmospheric parameter values, the analysis of line profile variations, and in the case of binaries, Fourier spectral disentangling and estimating the light factor $\alpha$. However, as we have seen, there is also still a lot of work to be done. When applying the LSD algorithm itself to do spectral disentangling, we currently suffer from degeneracy. And at this moment, we do not know how the algorithm performs for stars with a temperature $T_{\text {eff }}$ lower than $5000 \mathrm{~K}$ or higher than $10000 \mathrm{~K}$. It is clear that further testing of the LSD method is paramount, and anyone interested in collaborating on the subject is welcome to contact us.

We are also grateful to Conny Aerts (KU Leuven/University of Nijmegen), Oleg Kochukhov (Uppsala University) and Jonas Debosscher (KU Leuven), for the very helpful discussions.

\section{References}

Debosscher, J., Aerts, C., Tkachenko, A., et al., 2013, A\&A, 556, A56

Donati, J.-F., Semel, M., Carter, B.D., et al., 1997, MNRAS, 291, 658

Ilijić, S., Hensberge, H., Pavlovski, K., \& Freyhammer, L.M., 2004, ASP Conf. Ser., 318, 111

Kochukhov, O., Makaganiuk, V., \& Piskunov, N., 2010, A\&A, 524, A5

Kupka, F., Piskunov, N., Ryabchikova, T.A., et al., 1999, A\&AS, 138, 119

Lehmann, H., Tkachenko, A., Semaan, T., et al., 2011, A\&A, 526, A124

Piskunov, N., \& Kochukhov, O., 2002, A\&A, 381, 736

Rodríguez, E., Rolland, A., Garrido, R., et al., 1998, A\&A, 331, 171

Shulyak, D., Tsymbal, V., Ryabchikova, T., et al., 2004, A\&A, 428, 993

Tkachenko, A., Van Reeth, T., Tsymbal, V., et al., 2013, A\&A, 560, A37

Tsymbal, V., 1996, ASP Conf. Ser., 108, 198

Zima, W., 2008, Comm. Asteroseismol., 155, 17 